Braunsfurth, M.G. and Skeldon, A.C. and Juel, A. and Mullin, T. and Riley, D.S.

1997

MIMS EPrint: 2007.133

Manchester Institute for Mathematical Sciences

School of Mathematics

The University of Manchester

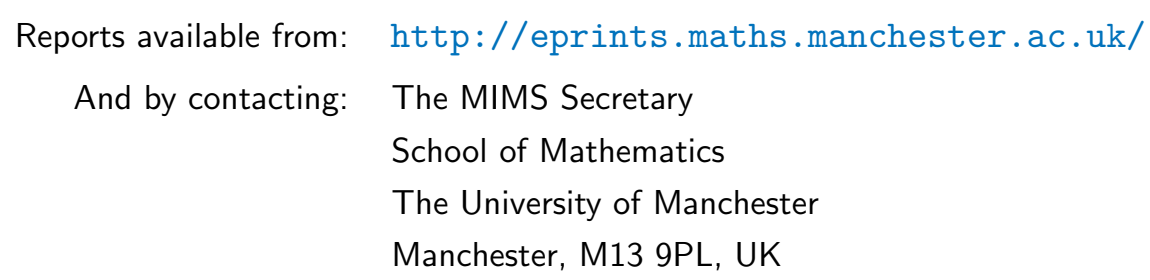

ISSN 1749-9097 


\title{
Free convection in liquid gallium
}

\author{
By M. G. BRAUNSFURTH ${ }^{1}$, A. C. SKELDON ${ }^{2} \dagger$, A. JUEL $L^{1}$, \\ T. MULLIN ${ }^{1}+$ AND D. S. RILEY $Y^{2}$ \\ ${ }^{1}$ Department of Atmospheric Oceanic and Planetary Physics, Clarendon Laboratory, \\ Oxford, OX1 3PU, UK \\ ${ }^{2}$ School of Mathematics, University of Bristol, Bristol, BS8 1TW, UK
}

(Received 22 November 1995 and in revised form 20 February 1997)

Free convection in liquid metals is of significant practical interest to the crystalgrowing community since the adverse effects of convective instabilities in the melt phase can be frozen into the solid product. Here, we present the results of a combined numerical and experimental study of steady convective flows in a sample of liquid gallium which is heated at one end and cooled at the other. Experimental measurements of temperature distributions in the flow are compared with the standard Hadley-cell solution and with the numerical results obtained from a two-dimensional model. Excellent quantitative agreement is found between all three for low Grashof numbers but a systematic divergence between the results is seen as this parameter is increased.

\section{Introduction}

The homogeneity and purity of monolithic crystals can be adversely affected by convective fluid motion in the melt. For example, in the horizontal Bridgman technique, layers of impurities known as striations degrade the performance of semiconductor devices made from the resulting solid. This is discussed by Müller \& Wiehelm (1964) who correlate the spacing of these striations in an InSb crystal with oscillations of the temperature in the liquid phase. Numerical work by Crochet, Geyling, \& van Schaftingen (1983) and a theoretical model developed by Thevenard et al. (1991) for crystals grown from a low-Prandtl-number fluid suggest that the striations are a result of periodic solidification and remelt at the interface.

An understanding of these important phenomena can best be achieved by first obtaining a deeper insight into the fundamental fluid mechanics underlying them. The aim of the present study is to investigate the details of the steady flows and thus provide a firm foundation for future work on the more complicated dynamical phenomena found at large temperature differences. These include thermal oscillations of the type discussed above. We present the results of a carefully controlled experimental study of the steady convective flows in a cavity containing molten gallium, which is a liquid metal above $302.8 \mathrm{~K}$. The fluid container is heated from one end and cooled at

$\dagger$ Present address: Department of Mathematics, City University, Northampton Square, London, EC1V OHB, UK.

$\ddagger$ Present address: Schuster Laboratory, The University of Manchester, Manchester M13 9PL, UK.

$\S$ Present address: Department of Theoretical Mechanics, University Park, Nottingham, NG7 2RD, UK. 


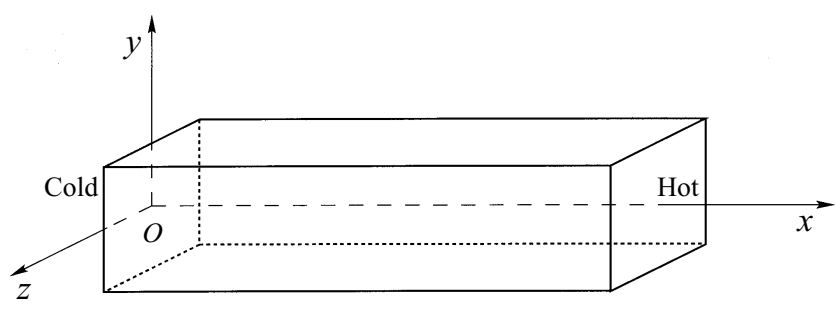

FIGURE 1. Geometry of the cavity.

the other and is designed to be a laboratory model of the Bridgman crystal-growing geometry, see figure 1. We compare observations from this experiment with theory and with the results of two-dimensional numerical calculations.

The non-dimensional parameters governing the flow are $P r$ and $G r$, the Prandtl and Grashof numbers, as well as the geometrical aspect ratios $A_{l}=l / h$ and $A_{w}=w / h$, where $l, w$ and $h$ are the length, width and height of the cell, respectively. The Grashof number, the primary parameter of interest here, can be changed in a controlled way by altering the applied temperature difference. In a given experiment, the Prandtl number is usually considered to be a constant, but it can be systematically varied by exploiting the temperature dependence of the material properties. This feature is used by Braunsfurth \& Mullin (1996) to explore the onset of oscillations and to uncover novel dynamical behaviour. Since molten gallium is a metal, its conductivity is large and its Prandtl number low, typically around 0.02 .

In previous experimental studies the main focus has been on time-dependent phenomena and there appear to be only two detailed experimental studies of steady flows in the published literature. In the first of these, Hurle, Jakeman \& Johnson (1974) report measurements of the motion in a cell with aspect ratio $A_{l} \times A_{w}=2.73 \times 1.18$. At the relatively large Grashof number of $10^{5}$, they find that the flow consists of a convection loop with fluid rising adjacent to the hot thermode and descending near the cold one (see figure 11a in Hurle et al.) This flow is three-dimensional in nature, and is asymmetric with respect to the midplane of the cell. In contrast, Hung $\&$ Andereck $(1988)$ use a cell with aspect ratio $1 \times 17.89 \times 17.78$ to generate an approximately two-dimensional flow. They show that a steady single convection loop loses stability to a longitudinal co-rotating multicellular state but report that direct observations of this phenomenon are difficult since the effect on the temperature field is very small.

A mathematical model relevant to this flow is discussed by Hart (1972) who obtains an exact parallel-flow result, often referred to as the Hadley solution, for an infinitely long layer of fluid with an applied horizontal temperature gradient. The stability of this solution to both transverse and longitudinal disturbances is considered by Hart (1972) and Hart (1983), and to longitudinal disturbances by Gill (1974). Hart shows that for Prandtl numbers above 0.015 , the first instability is to a longitudinal oscillatory solution. Hart's results are refined and extended by Laure (1987) and Kuo \& Korpella (1988), who show that for Prandtl numbers above 0.034 instability of the Hadley cell takes the form of a longitudinal oscillatory solution. For Prandtl numbers below 0.034 , the first transition occurs at a Grashof number of approximately $10^{4}$ and leads to a steady transverse roll solution instead. Laure (1987) performs a weakly nonlinear stability analysis and shows that a co-rotating roll solution is locally stable.

The effect of endwalls in a long cavity is considered by Cormack, Leal \& Imberger (1974) who use an asymptotic approach. This involves dividing the cavity into three 
regions: a central region consisting of a parallel flow and two approximately square end regions in which the flow turns round. Cormack et al. find that the Hadley model gives a reasonable solution to the flow in the centre of the cavity provided $\mathrm{Gr}^{2} \mathrm{Pr}^{2} / A_{l} \ll 3$. For the experimental configuration used in our study, this suggests that a parallel flow might be expected provided $G r \ll 5.5 \times 10^{4}$. Further theoretical progress with realistic boundary conditions and aspect ratios is difficult and hence recent studies has been made using numerical techniques.

Convection in molten gallium was used as a 'benchmark' problem for numerical methods and the results of the exercise are reported by Roux (1990). The main effort is focused on rectangular cavities with $A_{l}=4$ and Prandtl numbers of 0 and 0.015 . The boundary conditions relevant to our experiment are non-slip, impermeable boundaries where the upper and lower walls are insulating and the endwalls are isothermal. In this case, the reported two-dimensional numerical results show that the flow is parallel over much of the cavity for low Grashof numbers. As the Grashof number increases the flow evolves first to a more pronounced single-cell state and then to one with three co-rotating cells. Eventually, the steady flow loses stability to transverse oscillations; the possibility of instability to longitudinal oscillations is not explored. This sequence is qualitatively the same at a Prandtl number of both 0 and 0.015 , although a change in the Prandtl number from 0 to 0.015 increases the critical Grashof number for the onset of oscillations from $2.5 \times 10^{4}$ to $3.8 \times 10^{4}$ (see also Winters 1988 for further results on $A_{l}=4$ cavities).

There has been a limited number of three-dimensional numerical studies. For a $1 \times 4 \times 1$ cavity and $P r=0$, both Afrid \& Zebib (1990) and Gervasio (see Roux 1990) find that the flow remains in a steady unicellular state for $G r<10^{5}$, but thereafter begins to oscillate. Gervasio and Extremet et al. (see Roux 1990) find that an increase in the Prandtl number to 0.015 inhibits the onset of oscillations so that the flow is steady for $\mathrm{Gr}<1.5 \times 10^{5}$.

Despite all of the above research, fundamental issues concerning the convective motion remain unresolved. Progress is difficult because full three-dimensional numerical studies are computationally expensive and also relevant experiments are difficult to perform in a carefully controlled way. The approach we adopt is to test the range of validity of the simple model flows by performing quantitative comparison between the results of established theory, numerical calculation and experiment. Here, we begin by considering the Hadley cell which, in the past, has provided the main source of theoretical results for comparing with experiment. Since this is an infinite-layer model, it is important to understand the range of validity of the approximation in bounded containers. We give the equations of motion and briefly review the Hadley model in $\S 2$. In $\S 3$, we discuss the numerical solution method and present computed results for parameter ranges relevant to the experiment. The experimental apparatus is described in $\S 4$. In $\S 5$, we discuss the experimental boundary conditions and the material properties of gallium and in $\S 6$ we compare the theory and the numerical solutions with experimental measurements. Finally, we present some conclusions in $\S 7$.

\section{The equations of motion}

The numerical model consists of a rectangular, fluid-filled cavity of height $h$ and length $l$. The fluid motion is driven by differential heating, the endwalls being held at constant temperatures $T_{h}, T_{c}$ where the suffices $h$ and $c$ correspond to hot and cold, respectively. As discussed in $\S 5.1$ below, the experimental upper and lower boundaries 
are, to a good approximation, insulating and the end boundaries are isothermal. All the walls are taken to be impermeable and non-slip.

The equations of motion are the Navier-Stokes equations coupled with the energy equation. For the range of temperatures appropriate to the experimental conditions the Boussinesq approximation is assumed to hold and hence, in the usual notation,

$$
\left.\begin{array}{rl}
\nabla \cdot \boldsymbol{u} & =0 \\
\frac{\partial \boldsymbol{u}}{\partial t}+\boldsymbol{u} \cdot \nabla \boldsymbol{u} & =-\frac{1}{\rho_{0}} \nabla p+v \nabla^{2} \boldsymbol{u}+g \beta\left(T-T_{c}\right) \boldsymbol{j} \\
\frac{\partial T}{\partial t}+\boldsymbol{u} \cdot \nabla T & =\kappa \nabla^{2} T
\end{array}\right\}
$$

with boundary conditions

$$
\boldsymbol{u}=0 \quad \text { and } \quad \frac{\partial T}{\partial y}=0 \quad \text { on } \quad y= \pm \frac{h}{2}
$$

and

$$
\boldsymbol{u}=0 \quad \text { and } \quad T=T_{h} \quad \text { on } \quad x=l, \quad \boldsymbol{u}=0 \quad \text { and } \quad T=T_{c} \quad \text { on } \quad x=0 .
$$

As pointed out by Gill (1966), equations (2.1) with these boundary conditions are centro-symmetric.

Non-dimensionalizing equations (2.1) using $h, v G r^{1 / 2} / h, h^{2} / v, G r \rho_{0} v^{2} / h^{2}$, as the length, velocity, time and pressure scales, respectively, and measuring temperature relative to $T_{c}$ in units of $\gamma=\left(T_{h}-T_{c}\right) h / l$ gives the non-dimensional equations

$$
\left.\begin{array}{rl}
\nabla \cdot \boldsymbol{u} & =0, \\
\frac{\partial \boldsymbol{u}}{\partial t}+G r^{1 / 2} \boldsymbol{u} \nabla \cdot \boldsymbol{u} & =-G r^{1 / 2} \nabla p+\nabla^{2} \boldsymbol{u}+G r^{1 / 2} T \boldsymbol{j}, \\
\frac{\partial T}{\partial t}+G r^{1 / 2} \boldsymbol{u} \cdot \nabla T & =P r^{-1} \nabla^{2} T,
\end{array}\right\}
$$

with the boundary conditions

$$
\boldsymbol{u}=0 \quad \text { and } \quad \frac{\partial T}{\partial y}=0 \quad \text { on } \quad y= \pm \frac{1}{2}
$$

and

$$
\boldsymbol{u}=0 \quad \text { and } \quad T=A_{l} \quad \text { on } \quad x=A_{l}, \quad \boldsymbol{u}=0, \quad \text { and } \quad T=0 \quad \text { on } \quad x=0 .
$$

The dimensionless groups are the Prandtl number, $\operatorname{Pr}=v / \kappa$, and the Grashof number, $G r=g \beta h^{3} \gamma / v^{2}$, where $v$ is the kinematic viscosity, $\kappa$ is the thermal diffusivity, $\beta$ is the coefficient of thermal expansivity, $g$ is the gravitational constant and $\rho_{0}$ is the density.

In the case of an infinitely long container with a constant horizontal thermal flux, a steady parallel-flow solution, known as the Hadley-cell solution, Hart (1972), exists to equations $(2.2)$ :

$$
\left.\begin{array}{rl}
u & =\frac{1}{24} G r^{1 / 2}\left(4 y^{2}-1\right) y, \\
v & =0, \\
T & =x+\frac{1}{24} \operatorname{Pr} G r\left(\frac{y^{4}}{5}-\frac{y^{2}}{6}+\frac{1}{16}\right) y .
\end{array}\right\}
$$




\section{Numerical techniques}

The aim of the numerical study is to compute the two-dimensional steady-state solutions for the parameter values relevant to the experiment so that a direct comparison can be made between the two sets of results. We used a finite element method applied to the pressure-integrated weak form of equations (2.2), that is

$$
\begin{aligned}
& \int_{\Omega}\left\{f_{u}\left[\frac{\partial u}{\partial t}+G r^{1 / 2}\left(u \frac{\partial u}{\partial x}+v \frac{\partial u}{\partial y}\right)\right]-G r^{1 / 2} p \frac{\partial f_{u}}{\partial x}+\frac{\partial f_{u}}{\partial x} \frac{\partial u}{\partial x}+\frac{\partial f_{u}}{\partial y} \frac{\partial u}{\partial y}\right. \\
& +f_{v}\left[\frac{\partial v}{\partial t}+G r^{1 / 2}\left(u \frac{\partial v}{\partial x}+v \frac{\partial v}{\partial y}-T\right)\right]-G r^{1 / 2} p \frac{\partial f_{v}}{\partial y}+\frac{\partial f_{v}}{\partial x} \frac{\partial v}{\partial x}+\frac{\partial f_{v}}{\partial y} \frac{\partial v}{\partial y} \\
& +f_{T}\left[\frac{\partial T}{\partial t}+G r^{1 / 2}\left(u \frac{\partial T}{\partial x}+v \frac{\partial T}{\partial y}\right)\right]+\operatorname{Pr}^{-1}\left(\frac{\partial f_{T}}{\partial x} \frac{\partial T}{\partial x}+\frac{\partial f_{T}}{\partial y} \frac{\partial T}{\partial y}\right) \\
& \left.+f_{p}\left(\frac{\partial u}{\partial x}+\frac{\partial v}{\partial y}\right)\right\} \mathrm{d} x \mathrm{~d} y=0 .
\end{aligned}
$$

Here $f_{u}, f_{v}, f_{T}$ and $f_{p}$ are test functions which are chosen so that there is no contribution from boundary integrals. The temperature and velocities are represented as a sum of quadratic basis functions whilst linear discontinuous basis functions are used for the pressure. A standard Galerkin formulation of the finite element method was adopted in which the test functions are chosen from the same set as the basis functions.

The calculations were carried out on a rectangular domain divided into quadrilateral nine-node elements. In order to capture any rapid changes at the boundaries of the cavity, the spacing of the nodes was graduated so that there were more elements near the edges. Results were computed with a grid of $30 \times 12$ elements which was further refined to $64 \times 24$ elements to test for numerical accuracy. The values for the magnitude of the temperature field in the centre of the cavity agree to within $1 \%$ for the two grids over the range of Grashof numbers considered here.

The resulting discretized equations are of the form

$$
\boldsymbol{M} \frac{\partial \boldsymbol{x}}{\partial t}+\boldsymbol{f}(\mathbf{x}, \boldsymbol{\alpha})=0 .
$$

Here, $\boldsymbol{M}$ is the mass matrix, $\boldsymbol{x}$ is the solution vector and $\boldsymbol{\alpha}$ is the vector of the control parameters, namely the Prandtl number, the Grashof number and the aspect ratio. Steady-state solutions to (3.2) are sought by solving

$$
f(x, \alpha)=0
$$

using Newton's method. This method is guaranteed to converge providing a good initial guess to the solution is given. For low Grashof numbers, we found $\boldsymbol{x}=0$ to be sufficient. A path-following approach was used to step along a branch as $\mathrm{Gr}$ is increased. All computed solutions were steady, but not necessarily stable. In principle, the linear stability of the solutions can be tested by finding the eigenvalues of the Jacobian of $\boldsymbol{f}$, but this is computationally expensive. Instead, to monitor for simple bifurcations, we evaluated the sign of the determinant of the Jacobian for each solution. Since Newton's method involves the calculation of the Jacobian, and computing the determinant is inexpensive, this provides a convenient indicator of steady-state transitions. However, as found in the GAMM workshop (Roux 1990), steady solutions to equations (2.2) can also lose stability at a Hopf bifurcation where a complex conjugate pair of eigenvalues crosses the imaginary axis. There is no 
(a)

(i)

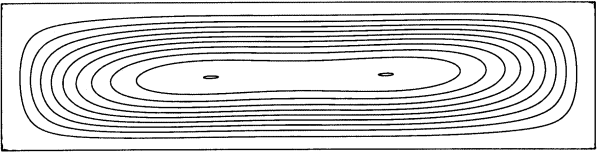

(ii)

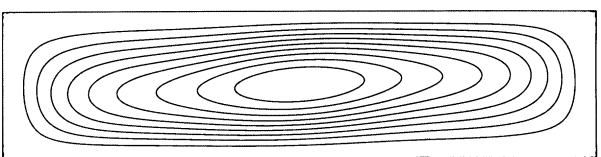

(iii)

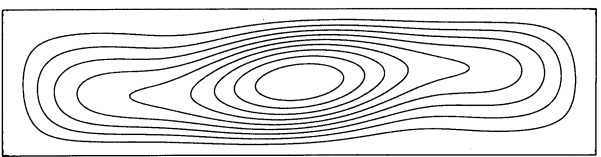

(iv)

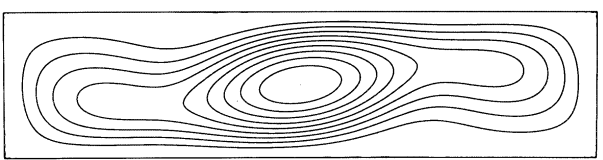

(b)

(i)

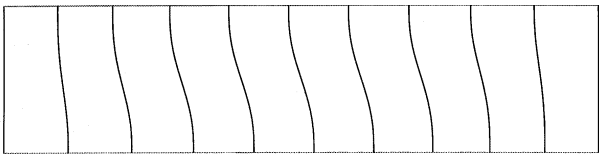

(ii)

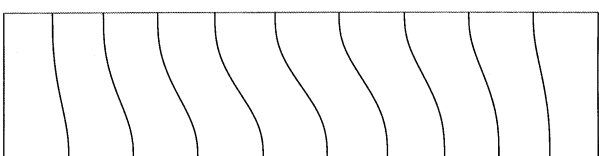

(iii)

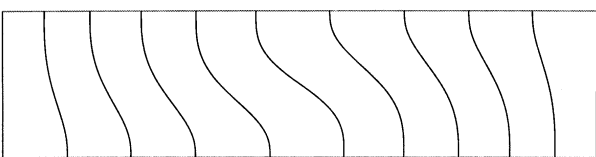

(iv)

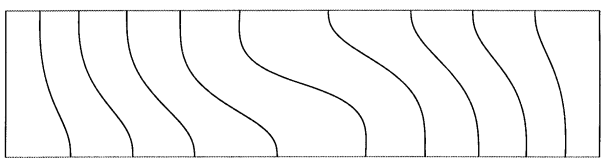

(c)

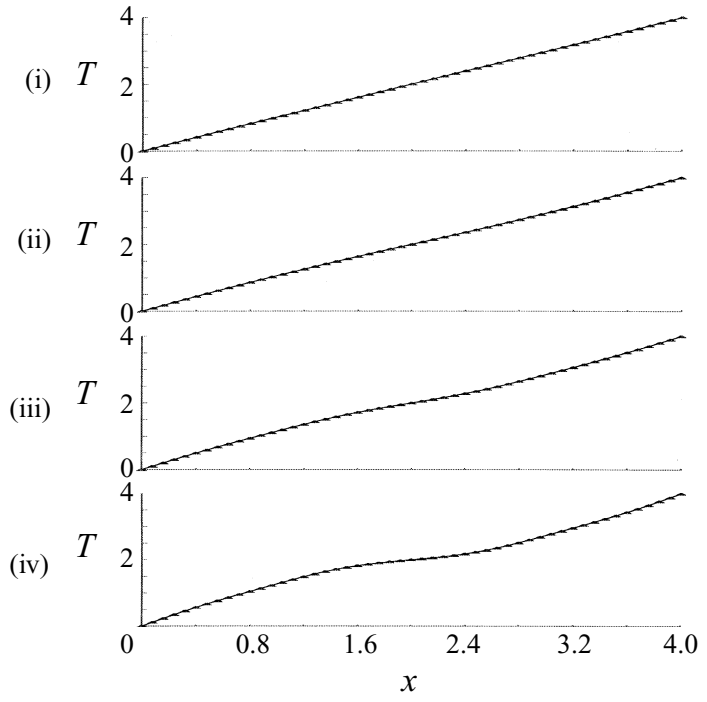

FIGURE 2. Computed results for a Prandtl number of 0.025 and a sequence of Grashof numbers: (a) streamlines, (b) isotherms, (c) horizontal temperature profiles along the centre of the cell. (i) $G r=5 \times 10^{3}$, (ii) $G r=1.0 \times 10^{4}$, (iii) $G r=2.0 \times 10^{4}$, (iv) $G r=4.0 \times 10^{4}$.

equivalent monitor for such bifurcations. In order to reduce the computational cost of finding such transitions, and hence establish the stability of a computed steady solution, we computed only the 'most dangerous' eigenvalues, i.e. the eigenvalues which have smallest real part (see Cliffe, Garratt \& Spence 1993).

Typical results for a Prandtl number of 0.025 and $A_{l}=4$ are shown in figure 2 as a sequence of streamlines, isotherms and horizontal temperature profiles for increasing Grashof number. At low Grashof number (see figure 2ai) the flow is parallel over much of the centre of the cavity and turns around in an approximately square end region, as in Cormack et al. (1974). As the Grashof number is increased, the region over which the motion is parallel shrinks and the flow can be approximated by the 
weakly nonlinear solution which Laure (1987) found to bifurcate from the Hadley-cell solution. An infinite layer has translational symmetry which is lost at the transition to co-rotating cells and hence a bifurcation occurs. The finite box of our two-dimensional calculations does not have this translational symmetry and thus this bifurcation is unfolded to give a smooth transition from parallel flow to rolls. In our case, the box contains only one of these rolls. As the Grashof number is increased further (figures 2aii-iv) the central 'eye' of the roll becomes more and more pronounced. For lower Prandtl numbers, an evolution to three co-rotating cells is observed in all the numerical studies reported in the GAMM workshop (Roux 1990), but this evolution is inhibited at the Prandtl numbers relevant to our case. Note that all the streamline patterns shown in figure 2 exhibit the centro-symmetry of the original equations.

It can be seen from the isotherms shown in figure $2(b)$ that extracting flow information from an experimental measurement of the temperature field is difficult. When the Prandtl number is zero, the diffusion equation governing the heat transfer is independent of the flow and the isotherms are equally spaced vertical lines. All heat is transferred by conduction in such a case, and a measure of the temperature field would give no information about the flow. In practice, gallium has a small, non-zero Prandtl number which couples the energy equation weakly to the flow. At low Grashof numbers, the flow is weak and the isotherms are only slightly perturbed from the vertical (see figure 2bi). As the Grashof number is increased, see figures 2(b)(ii-iv), the isotherms become more and more deformed. However, even at the highest Grashof number of $4 \times 10^{4}$, the variation at the centre of the cavity is less than $20 \%$ of the temperature difference between the two ends. The weak effect of the flow on the temperature field is further emphasized by the horizontal temperature profiles along the centre of the cell, shown in figure 2(c). If all heat transfer were by conduction, these profiles would be linear. Only very small variations from linearity are evident in figure 2(c)(i, ii). However, as the Grashof number is increased the temperature profiles become horizontal in the centre of the cell, i.e. in the middle of the 'eye', as can be seen in figure 2(c)(iii, iv).

Calculations of the most dangerous eigenvalues show that the computed solutions are stable to two-dimensional disturbances. For the same boundary conditions and aspect ratio, and Prandtl numbers of 0 and 0.015 , contributors to the GAMM workshop (Roux 1990) find that transverse oscillations occur. Winters (1988) finds that, in an aspect-ratio- 4 cavity, the onset of oscillations occurs at a Hopf bifurcation at a Grashof number of $2.55 \times 10^{4}$ for a Prandtl number of 0 , and of $3.8 \times 10^{4}$ for a Prandtl number of 0.015 . Increasing the Prandtl number to 0.025 further inhibits the onset of oscillations, see Skeldon, Riley \& Cliffe (1996) who find no evidence of a Hopf bifurcation for Grashof numbers up to $1.5 \times 10^{5}$.

\section{The experiment}

\subsection{Experimental apparatus}

A schematic of the experimental apparatus is presented in figure 3. We outline only the essential features of the apparatus here; the interested reader is referred to Braunsfurth \& Mullin (1996) for further details.

The working section of the experiment consists of a ceramic boat of rectangular cross-section which holds the liquid gallium between two end plates, see figure 3 . Two boats of different lengths are used in separate sets of experiments. One is of length $51.2 \pm 0.1 \mathrm{~mm}$ and the second of length $38.7 \pm 0.1 \mathrm{~mm}$. Both boats have an inner 


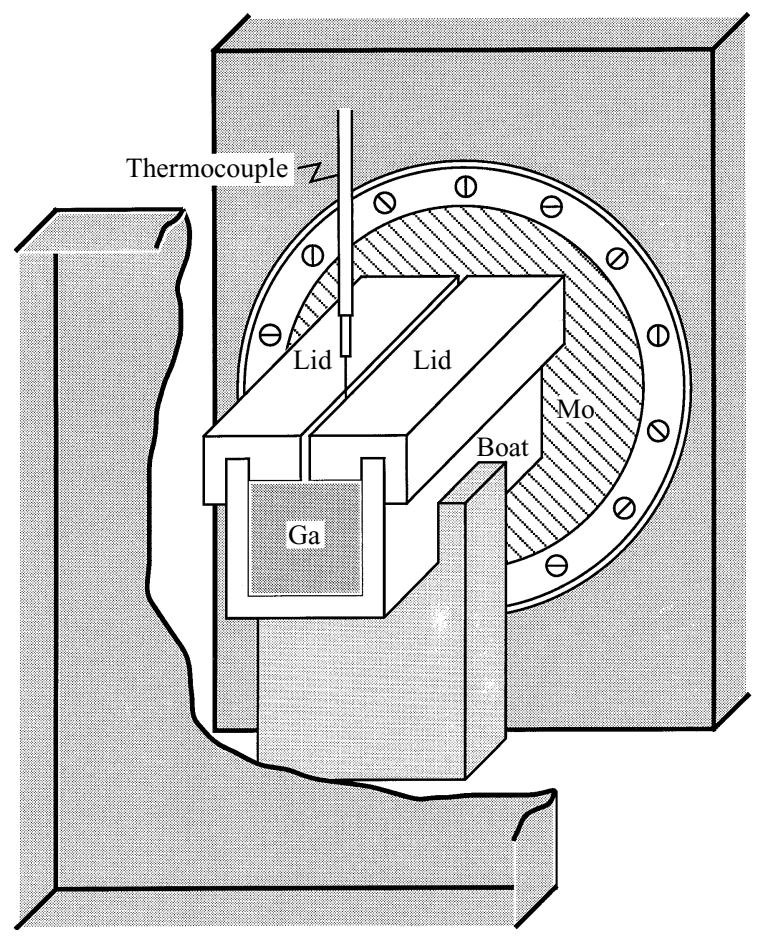

FIGURE 3. Schematic of the experimental apparatus.

width $12.82 \pm 0.1 \mathrm{~mm}$ and a height of $12.81 \pm 0.1 \mathrm{~mm}$, giving chambers of dimensions $1.00 \times 3.99 \times 1.00$ and $1.00 \times 3.02 \times 1.00$, respectively. Each boat can be covered by a lid which makes contact with the gallium.

The temperature gradient was applied to the liquid gallium by heating and cooling the metal walls which form the ends of the boat. In order to achieve high-quality repeatable results every effort was made to ensure that spatially uniform heating and cooling was applied at the molybdenum boundaries. Moreover, good temporal stability was required on both short and long time scales, since many of the experimental observations were taken over periods of several days. In order to achieve this, temperature-controlled fluid was circulated through large copper boxes containing 0.71 of liquid. This volume had sufficient thermal inertia to act as a low-pass filter, damping out external temperature fluctuations.

Measurements of the temperatures at different points in the gallium were taken using two sheathed and insulated K-type (chromel-alumel) thermocouples. There is a thin slot along the length of the lid so that a measuring thermocouple can be inserted into the melt. The width of the slot is $0.5 \pm 0.1 \mathrm{~mm}$, that is $4 \%$ of the width of the container. The outer diameter of the thermocouple sheath was $250 \mu \mathrm{m}$, which is approximately $2 \%$ of the width of the channel so that the probe did not significantly block the flow. Furthermore, the largest Reynolds number for the flow around the probe was less than 4 , well below the accepted critical Reynolds number for vortex shedding from the probe.

The thermocouple was mounted on a micro-manipulator which was driven by two computer-controlled stepping motors. This enabled the positioning of the thermocouple to an accuracy of $\pm 0.1 \mathrm{~mm}$ in each direction. The signal was processed using a thermocouple amplifier and sampled using a 12-bit analog-to-digital converter. The 
data were stored and analysed using a personal computer. This computer was also used both to control the stepper motors which located the thermocouples, and to fix the settings of the external temperature controllers which provided the temperature gradient along the sample of gallium.

\subsection{Experimental measuring techniques}

Vertical and horizontal scans of the gallium were made with the thermocouples, allowing temperature profiles to be constructed. The computer control allowed repeatable positioning of the thermocouples to within 1 part in 304. Each time the probe was moved the flow was allowed to settle before measurements were made. The settling time was empirically determined to be $60 \mathrm{~s}$ if the movement was a small vertical shift, but $1000 \mathrm{~s}$ if the probe was moved sideways. At each position, samples of between 600 and 1000 data points were taken over a period of minutes and the mean, minimum and maximum values were recorded. The minimum and maximum values give an indication of the size of fluctuations and measurement noise, and thus provide an estimate of the experimental uncertainty. Each scan was made in first one direction and then the other. The resulting pairs of profiles could be superimposed showing the repeatability and robustness of the experimental measurements.

When making vertical temperature scans it was necessary to ensure that the tip of the thermocouple did not collide with the base of the pyrophyllite channel. This was achieved by using a plastic needle held in the micromanipulator to find the bottom of the container. The origin of the temperature axis was set at the mid-height of the gallium layer, in the centre of the cavity, thus allowing a compensation for an unknown offset which arises from an arbitrary shift in the measuring electronics. The temperature was scaled by $\left(T_{h}-T_{c}\right) / A_{l}$ where the temperature difference $T_{h}-T_{c}$ was obtained from the calibration curve discussed below.

The majority of the experimental measurements were taken down a vertical line in the centre of the cavity, i.e. halfway between the ends and sidewalls. If the transport of heat were due entirely to conduction, the measured temperature would be independent of height. However, there was a weak convective flow and a small but discernible temperature dependence existed which increased as the Grashof number was raised. Thus the vertical temperature profiles provided a measure of the strength of the convective flows. In particular, the vertical temperature difference between $y=0$ and $y=0.5$ was extracted from each profile and it is this measure which is used in the quantitative comparison between the observations and the calculations. The upper and lower error bars on each point were estimated by considering the values of the minimum and maximum temperatures averaged over the entire profile.

Most of the horizontal measurements were made along the centreline of the cavity with the probe located at half the height of the fluid layer. These profiles were used to study the lengthwise structure of the convective flow.

\section{Boundary conditions and material properties}

In order for quantitative comparison to be made between the experiment, theory and numerics, two key issues need to be addressed. First, the appropriate boundary conditions have to be considered. Secondly, the material properties of gallium are, perhaps surprisingly, not well-known. The latter can introduce a systematic error in the conversion of the data from dimensional to non-dimensional form and we discuss below how we take this into account. 


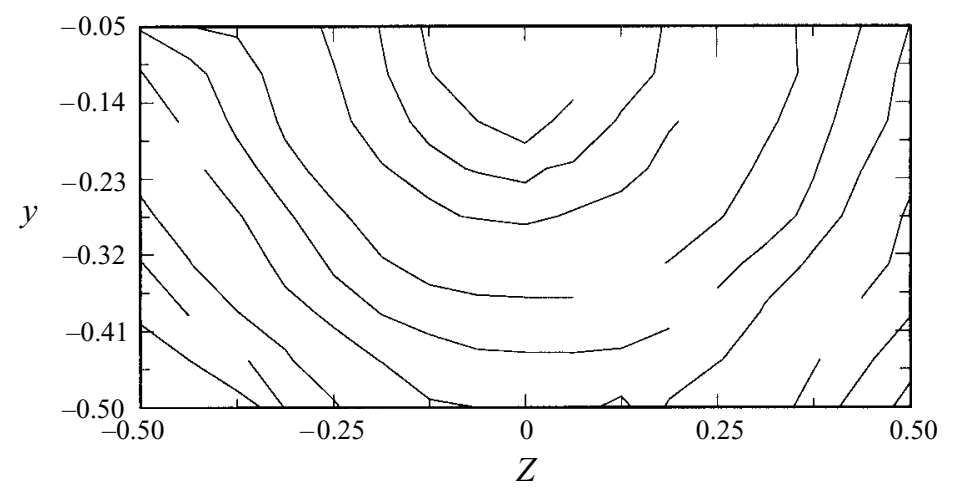

FIGURE 4. Boundary conditions at the cold end.

\subsection{Boundary conditions}

We assume that the non-slip boundary conditions are satisfied along all the bounding surfaces since the gallium was in contact with rigid walls. When the lid is present this condition also holds on the top surface for all but the narrow region of the slot. Indeed, measurements taken both with and without the lid present indicate that the qualitative nature of the flow is robust as discussed by Braunsfurth (1994) but there are small quantitative differences. In contrast, theoretical and numerical work suggest a marked difference between a stress-free boundary and a non-slip impermeable boundary (see, for example, Kuo \& Korpela 1988, Roux 1990). However, we find that the experimental boundary conditions are effectively always rigid due to the rapid formation of an oxide skin on the top surface of the gallium when exposed to air. Of course the thermal properties of the oxide layer are not the same as the lid and presumably this, along with small effects such as variations in the thickness of the oxide layer, lead to the small quantitative differences between the results taken with and without a lid.

Every effort was made to achieve good thermal control. The base and lateral walls of the boat are made from pyrophyllite, and the lid from MACOR. These have thermal conductivities of $1.6 \mathrm{~W} \mathrm{~m}^{-1} \mathrm{~K}^{-1}$ and $1.48 \mathrm{~W} \mathrm{~m}^{-1} \mathrm{~K}^{-1}$, respectively, which are 18 and 29 times lower than that of gallium in the operating range of the experiment. In addition, each wall is approximately $5 \mathrm{~mm}$ thick and we therefore consider them to be good practical approximations to insulators.

The uniformity of the temperature distribution at the molybdenum endwalls was investigated by bending a thermocouple probe so that the tip was in contact with the metal wall. Then, temperature measurements were taken on an $11 \times 11$ grid of points. Typical results for the cold end for the channel of dimensions $1.00 \times 3.02 \times 1.00$ are presented in figure 4, which shows the temperature distribution in contour form over the bottom half of the endwall. The sidewalls of the container are at the nondimensionalized positions of \pm 0.5 , which correspond to the edges of the graphs. It was necessary to take all of these particular measurements without a lid and, as a result, the meniscus at the top surface and the corners of the boat have a significant effect. In particular, the point of contact between the meniscus and the wall is influenced by movement of the probe and varied by up to $2 \mathrm{~mm}$. For this reason, temperature measurements at the upper half of the wall are unreliable and we present only those taken in the lower half. We assume these results approximately mirror those in the upper half of the cell when the lid is present. 


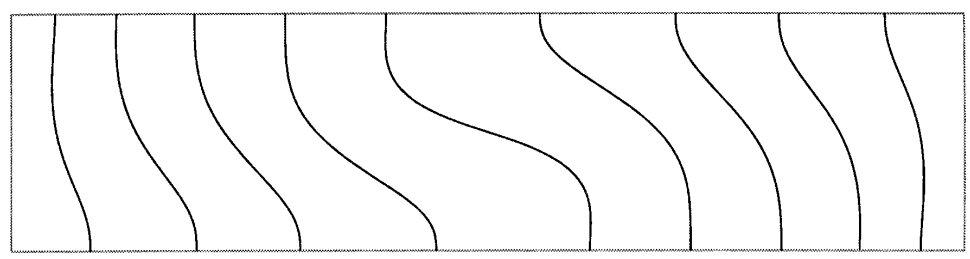

FIGURE 5. Isotherms for parabolic end temperature profile. $G r=4 \times 10^{4}$.

It can be seen in figure 4 that there is a rise in the temperature in the central region which is due to the finite thermal diffusivity of the metal endwalls. At the centre more heat is delivered due to the additional heat transport through the gallium by convection. At the hot end, we find the temperature at the centre of the wall to be correspondingly lower. The amount of heat transferred by convection increases as the Grashof number increases and so this effect is more pronounced the higher the applied temperature difference driving the flow. The results presented in figure 4 are for a relatively high applied temperature difference of $14 \mathrm{~K}$ across the melt. At this temperature the amplitude of the variation of the temperature across the metal endwalls is $1.6 \mathrm{~K}$. Although this variation in temperature at the endwalls does not conform to the isothermal boundary conditions assumed for the numerical solutions, the effect is small and symmetric. In order to test whether this variation drives any significant extraneous secondary flows, computations were carried out with parabolic temperature profiles at the end boundaries to mimic more closely the experiment. The resulting streamlines are indistinguishable from those for the isothermal boundary conditions and the computed values for the temperature in the centre of the cavity change by less than $4 \%$. In figure 5 isotherms for a Grashof number of $4 \times 10^{4}$, at the upper end of the range under consideration here, are shown. This should be compared with figure $2(b)$ (iv). The presence of the parabolic end temperature profiles is just observable in the shape of the isotherms at the extremes of the cavity.

The prescribed measured temperature in the copper boxes is significantly different from the centreline temperature measured at the wall inside the gallium. We attribute this drop to a combination of the interface resistance between the silicone oil, molybdenum walls and liquid gallium. The experimental Grashof numbers are calculated using the temperatures $T_{h}$ and $T_{c}$ measured on the centreline in the gallium at the endwalls. In order to relate this to the temperature set by the controllers, a calibration curve was established as discussed in detail in Braunsfurth (1994).

\subsection{Material properties}

The gallium used in the experiments was $99.99 \%$ pure. Results taken over a period of months are self-consistent, suggesting that any contamination which occurred over this period did not significantly change the material properties.

We collected available data on the material properties of gallium in the temperature range of the experiment from Brandes \& Brook (1992), Filyand \& Semenova (1968), Hampel (1954), Iida \& Guthrie (1993), Kaye \& Laby (1982), March (1968), Smithells \& Brandes (1976), the Metals Handbook (1990) and Touloukian et al. (1979). The melting point of gallium is $302.8 \mathrm{~K}$. Brandes \& Brooke give the following temperature dependence for the viscosity of gallium:

$$
\eta=0.4359 \exp \frac{481}{T} \mathrm{mN} \mathrm{s} \mathrm{m}^{-2}
$$

where $T$ is in Kelvin. The available viscosity measurements in the range of interest 


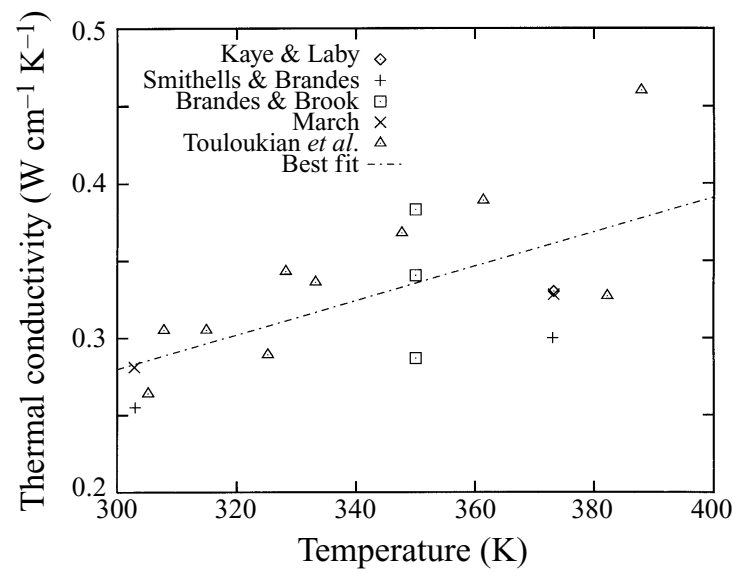

FIGURE 6. Temperature dependence of the thermal conductivity for liquid gallium for the temperature range of the experiment.

are in close agreement with this formula. We have been unable to find an equivalent formula for the temperature dependence of the specific heat of gallium. We considered the mean of the recorded values and found $c_{p}=0.36 \pm 0.03 \mathrm{~kJ} \mathrm{~kg}^{-1} \mathrm{~K}^{-1}$, where the error quoted is the standard deviation. Although this represents an uncertainty of $8 \%$, it is much smaller than the uncertainty in the thermal conductivity. We show all the available data for the thermal conductivity plotted in figure 6 as a function of $T$. From this, it can be seen that there is considerable scatter in the data. A linear least-squares fit suggests that

$$
k=(0.11 T-5) \pm 4 \mathrm{~W} \mathrm{~m}^{-1} \mathrm{~K}^{-1},
$$

and consequently there is a systematic error in the absolute value of the Prandtl number, $\operatorname{Pr}=v / \kappa=\eta c_{p} / k$, of about $16 \%$.

The density of gallium is known more accurately and Weast et al. (1993) quote the temperature dependence of the density as

$$
\rho=6.32723-7.3743 \times 10^{-4} \mathrm{~T}+1.37767 \times 10^{-7} \mathrm{~T}^{2} \mathrm{~g} \mathrm{~cm}^{-3} .
$$

This fits the available data extremely well, the error being less than $0.2 \%$. The coefficient of thermal expansivity, $\beta$, is less well-known and we have used the value given by Iida \& Guthrie (1993) of $1.3 \times 10^{-4} \mathrm{~K}^{-1}$ at $303 \mathrm{~K}$. When this is used together with the expressions (5.1), (5.3) for the viscosity and density, respectively, the Grashof number for the longer channel is given by

$$
G r=1.413 \times 10^{5} \times\left(1-2.357 \times 10^{-4} T\right) \exp \left(-\frac{962}{T}\right) \Delta T .
$$

Here terms of order $T^{2}$ and higher have been neglected as they produce negligible corrections for the temperatures under consideration.

It is assumed in the derivation of the mathematical model, given by equation (2.2), that the thermal conductivity and the viscosity are independent of temperature and that the temperature dependence of the density is only important in providing a buoyancy force to drive the flow. This approximation arises from expanding the physical parameters about a fixed reference temperature and neglecting the higher-order terms. When relating the experimental measurements to the theoretical/numerical 
results, the temperature $T$ in expressions (5.1),(5.2),(5.3) and subsequently (5.4) is the reference temperature. The choice of the actual reference temperature is not unique and we have used the mean temperature in the flow for all the comparisons so that there is consistency throughout.

\section{Results}

\subsection{Vertical flow structure}

The main comparison between the results of analytical theory, numerical calculation and experimental observation is made using the vertical profiles measured at the centre of the cavity, i.e. halfway between the hot and cold ends, and halfway between the sidewalls of the $3.99 \times 1.00 \times 1.00$ cavity.

Graphs comparing the experimental, analytical and numerical temperature profiles are presented in figure 7. The Prandtl number varies from $0.025 \pm 0.04$ for the lower Grashof numbers to $0.024 \pm 0.04$ for the higher Grashof numbers. The profile shown in figure $7(a)$ is for a Grashof number $G r=8.6 \times 10^{3}$, corresponding to a total temperature difference between the ends of $1.38 \mathrm{~K}$. The solid curve gives the Hadley-cell temperature dependence calculated from equation (2.3). The dotted curve represents the computed temperature profile in the centre of the cell, whilst the points are the experimental measurements. For this low Grashof number there is good agreement between the experimental data and the numerical and Hadley-cell solutions. There are large error bars on the experimental points because in this case the total amplitude of the temperature profile corresponds to $0.08 \mathrm{~K}$ which is close to the limit of the accuracy of the experiment. In addition, large amplification was used on the thermocouple signal and hence there is a significant contribution to the error from instrumentation noise. The flow consists of a large weak convective cell. The streamline plot for similar parameter values shown in figure 2(a)(ii) suggests that the flow is approximately parallel for the central third of the cavity.

The vertical profile at a larger Grashof number of $G r=1.3 \times 10^{4}$ is shown in figure $7(b)$. The agreement between the numerical calculation and the experimental data points is still very good for this Grashof number. However, both have diverged slightly from the analytical solution. The error bars on this graph appear much smaller than in figure $7(a)$, since the convective flow is now significant and the temperature signal is larger than the experimental noise. In addition, the temperature has been non-dimensionalized with the now-larger applied temperature difference. The corresponding numerical streamline plot of figure 2(a)(iii) suggests that the flow in the centre of the cavity is no longer parallel and that the endwalls have a significant effect.

At an even larger Grashof number of $G r=3.4 \times 10^{4}$, good qualitative agreement between the Hadley-cell, numerical and experimental profiles is still found, as shown in figure 7(c). However, the two-dimensional numerical solution has now diverged from the experimentally measured profile. We believe that this is due to the influence of the lateral walls so that three-dimensional effects are important in the experiments. Indeed recent numerical studies by A. Juel (1997, personal communication) have confirmed this, but a more detailed analysis needs to be performed before a definite statement can be made.

A more useful way of showing the dependence of the strength of the convective flow as a function of temperature difference is to plot the amplitude of the vertical temperature profiles against the Grashof number, as in figure 8 . The results suggest 

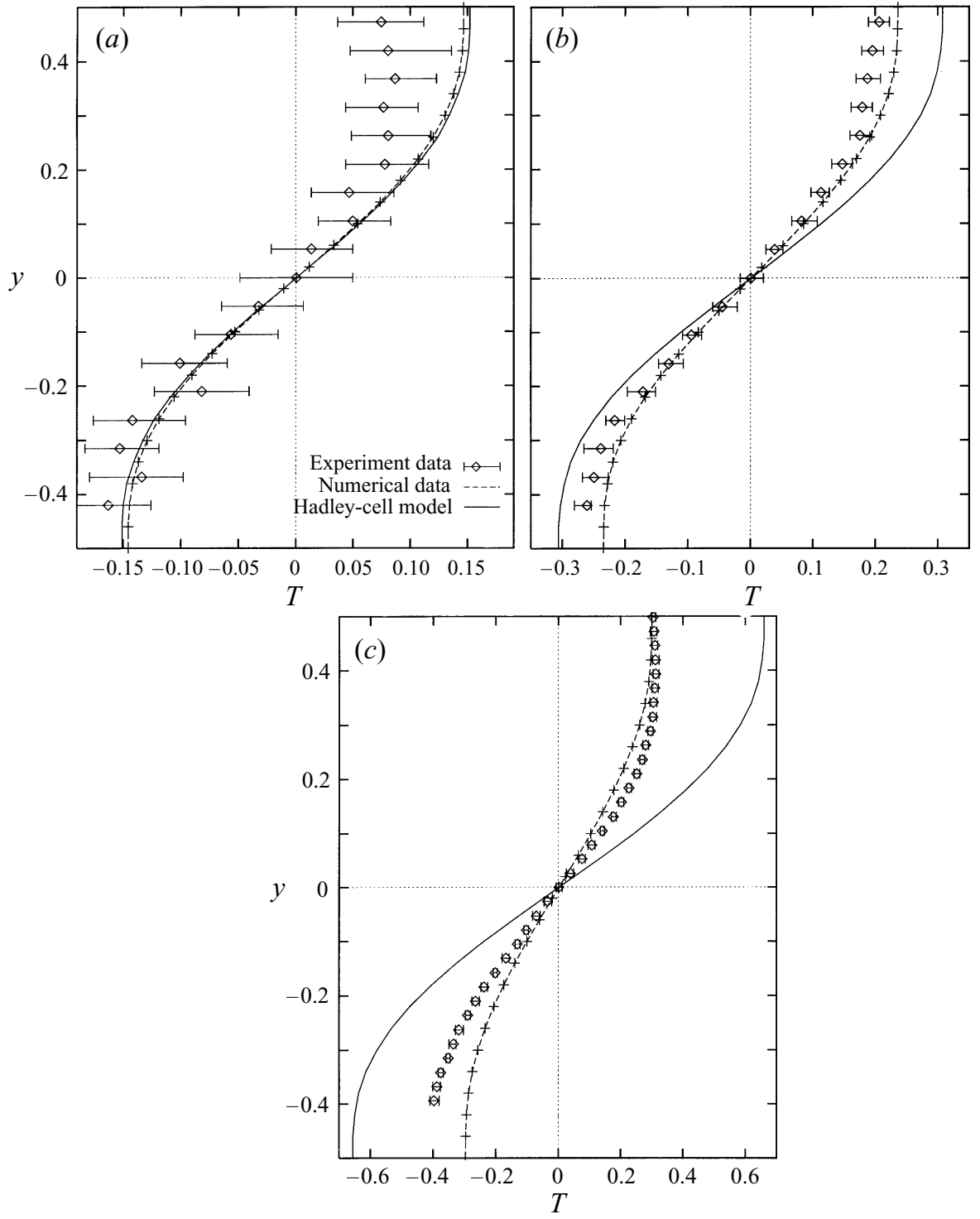

FIGURE 7. Experimentally measured vertical temperature profiles in the centre of the cavity compared with the two-dimensional calculations. (a) $\mathrm{Gr}=8.6 \times 10^{3}$, (b) $\mathrm{Gr}=1.3 \times 10^{4}$, (c) $\mathrm{Gr}=3.4 \times 10^{4}$.

that at low Grashof number, $G r<10^{4}$, the Hadley-cell solution gives a good approximation to the experimental flow. It is interesting to note that this upper limit is approximately the value of the Grashof number where, in the infinite layer, the parallel-flow solution loses stability to a flow with co-rotating rolls (see Laure 1987). For greater values of the Grashof number the two-dimensional numerical solution remains close to the experimental results but the two data sets diverge for $\mathrm{Gr}$ greater than a value of about $2 \times 10^{4}$. For values of the Grashof number above this the agreement becomes more qualitative than quantitative.

As discussed at the beginning of this section there is a large uncertainty in the value of the Prandtl number because of the lack of knowledge of the material constants of gallium. In figure 9 we display the experimental data superimposed on three separate 


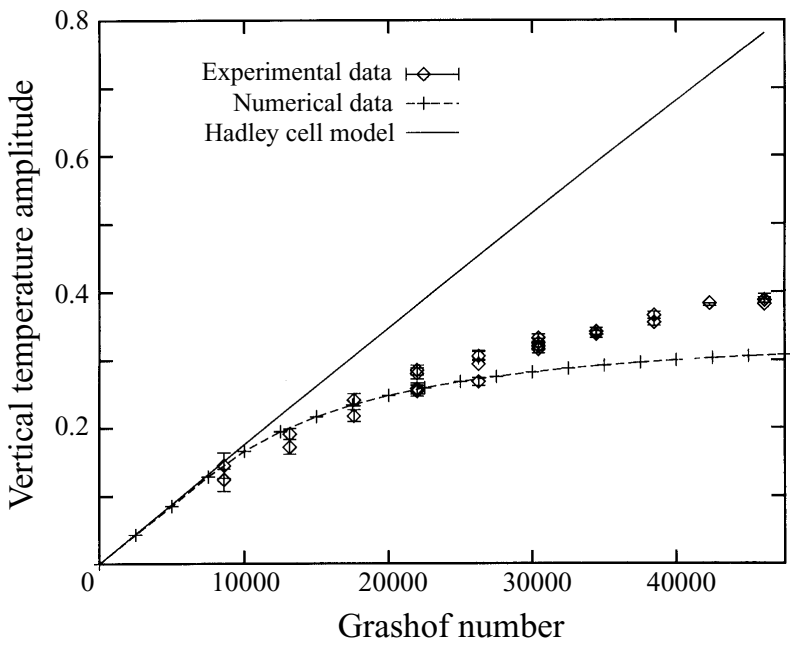

FiguRE 8 . The vertical temperature drop in the centre of the cavity as a function of the Grashof number for the Hadley model and the two-dimensional numerical results for $\operatorname{Pr}=0.025$ compared with the experimental data.

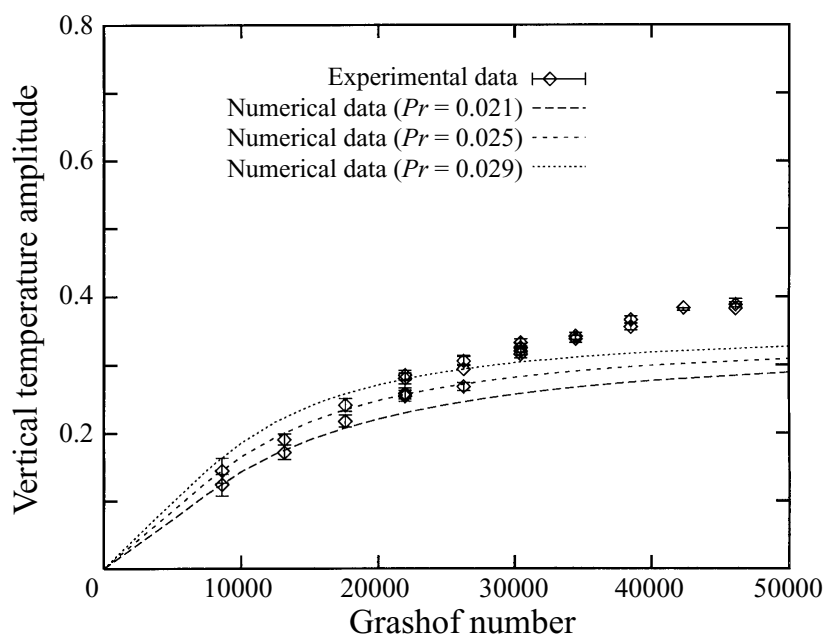

FIGURE 9. The vertical temperature drop in the centre of the cavity as a function of the Grashof number for Prandtl numbers $0.021,0.025$ and 0.029 for the Hadley model and the two-dimensional numerical results compared with the experimental data.

curves calculated using the two-dimensional numerical model with Prandtl numbers of $0.021,0.025$ and 0.029 . These correspond to the $\pm 16 \%$ systematic error in the Prandtl number referred to above. One obvious feature of the numerical curves is that their qualitative form is independent of $\mathrm{Pr}$ for the range studied. Also, at a fixed Grashof number, the vertical temperature drop is bigger for larger Prandtl numbers. However, the most important aspect of the intercomparison is that there remains a systematic divergence between the two data sets and thus the differences between the experimental and numerical results cannot be explained in terms of uncertainty in the Prandtl number.

All three temperature profiles presented in figure 7 show some evidence of asym- 

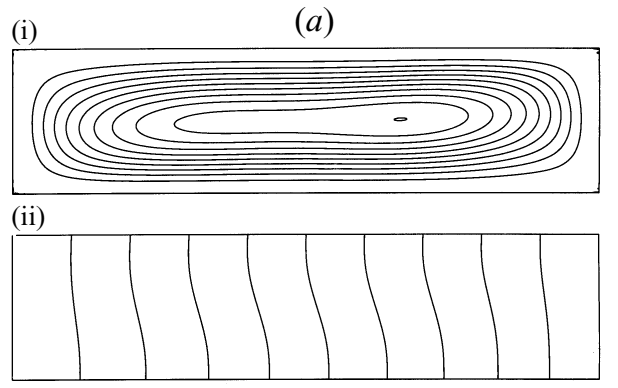

(iii)

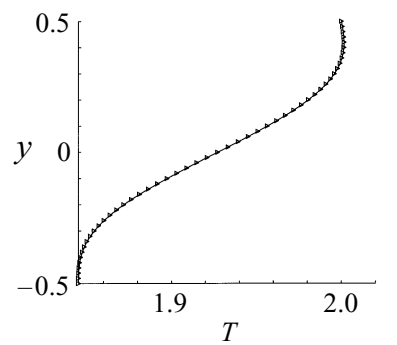

(i)

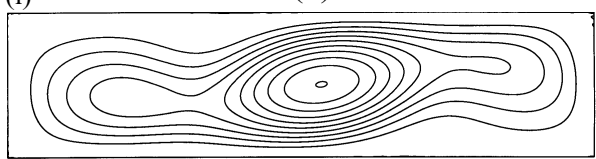

(ii)

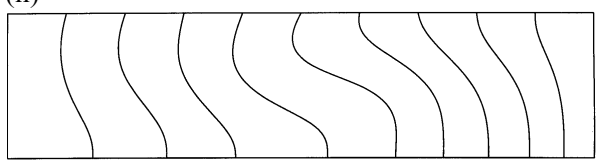

(iii)

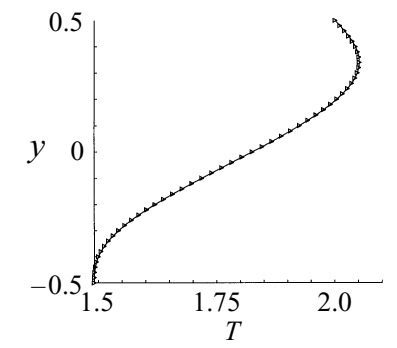

FIGURE 10. Numerical results for a rectangular cavity with a conducting upper boundary and an insulating lower boundary. (a) $G r=5 \times 10^{3}$, (b) $G r=4 \times 10^{4}$ : (i) streamlines, (ii) isotherms, (iii) vertical temperature profile in the centre of the cavity.

metry. This could be due to lack of symmetry in the boundary conditions or to non-Boussinesq effects; we have investigated the effect of both of these in our two-dimensional numerical model. Gray \& Giorgini (1976) systematically derive conditions for the validity of the Boussinesq approximation. In our case, based on the available information about the material properties, all their criteria are satisfied and we therefore expect any non-Boussinesq effects to be small. This was confirmed numerically by introducing terms into equations (2.2) to allow for both an exponential variation of the viscosity with temperature and a linear variation of the thermal conductivity with temperature as given by expressions (5.1) and (5.2) respectively.

In order to examine the effect of the boundary conditions we considered the extreme case of an upper conducting boundary whilst maintaining the lower one as an insulator. In figure $10(a)$ we show the streamlines, the isotherms and the corresponding vertical temperature profile in the centre of the cavity for the Grashof number of $5 \times 10^{3}$. The lack of centro-symmetry is obvious in the streamline plot shown in figure 10(a)(i), which can be compared directly with figure 2(a)(i). At this low Grashof number the convective flow is weak and this asymmetry is not so readily discernible from the isotherms, see figure 10(a)(ii). A slight reduction in the temperature at the top end of the vertical temperature profile is observable however in figure 10(a)(iii). At greater Grashof numbers these features become more prominent and when the value of $4 \times 10^{4}$ is reached, see figure $10(b)$, there is a clear qualitative difference between the shape of the vertical temperature profile in the conducting/insulating boundary case and that of the purely insulating one. The conducting upper boundary also leads to a drop in the amplitude of the vertical temperature. At a Grashof number of $4 \times 10^{4}$ the conducting/insulating case has a vertical temperature drop which is $15 \%$ lower than the corresponding insulating case. A plot of the amplitude of the vertical temperature drop versus Grashof number has the same qualitative shape as the numerical curve as shown in figure 8, with the slight difference that it is flattened at a lower vertical tem- 


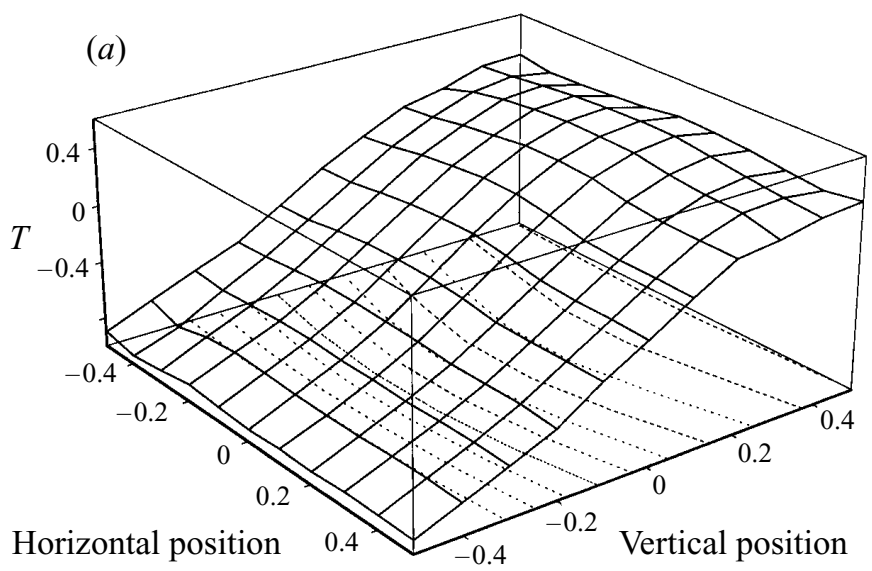

(b)

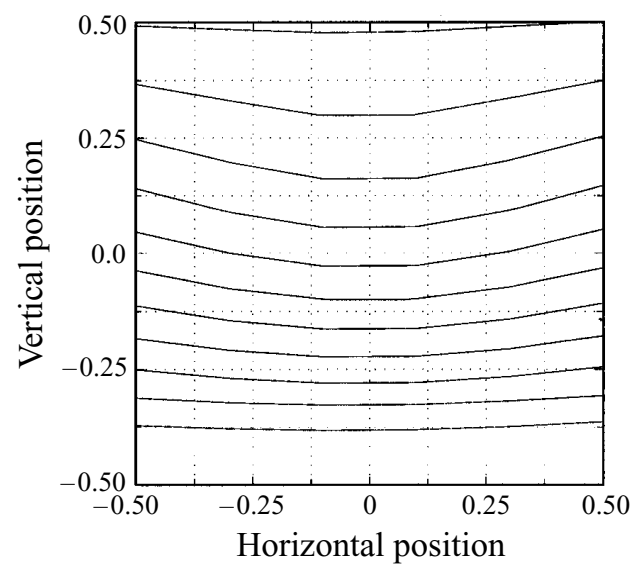

Figure 11. (a) Temperature surface, $(b)$ temperature contours on the cross-section at the centre of the cavity.

perature amplitude. In the experiment the upper and lower boundary conditions are neither perfectly insulating nor perfectly conducting. The qualitative shape and quantitative comparison of the vertical temperature amplitude against Grashof number suggest that insulating boundary conditions are, however, a reasonable approximation.

The good agreement between the two-dimensional numerical results and the experimental results suggests that any three-dimensional effects do not have a significant effect on the flow at the Grashof numbers considered here. In order to check this possibility more fully, measurements of the temperature on vertical cross-sections of the cavity were made.

\subsection{Crosswise flow structure}

In this set of experiments the applied temperature difference was set moderately high, at $\Delta T=24 \mathrm{~K}$, which corresponds to a Grashof number of $G r=1.1 \times 10^{5}$. The Prandtl number is $\mathrm{Pr}=0.024$ and the aspect ratio is $1.00 \times 3.02 \times 1.00$.

The temperature distribution is displayed as a function of horizontal and vertical position in figure 11(a), and is constructed from a set of temperature measurements taken halfway between the hot and cold ends of the boat. The corresponding contour 

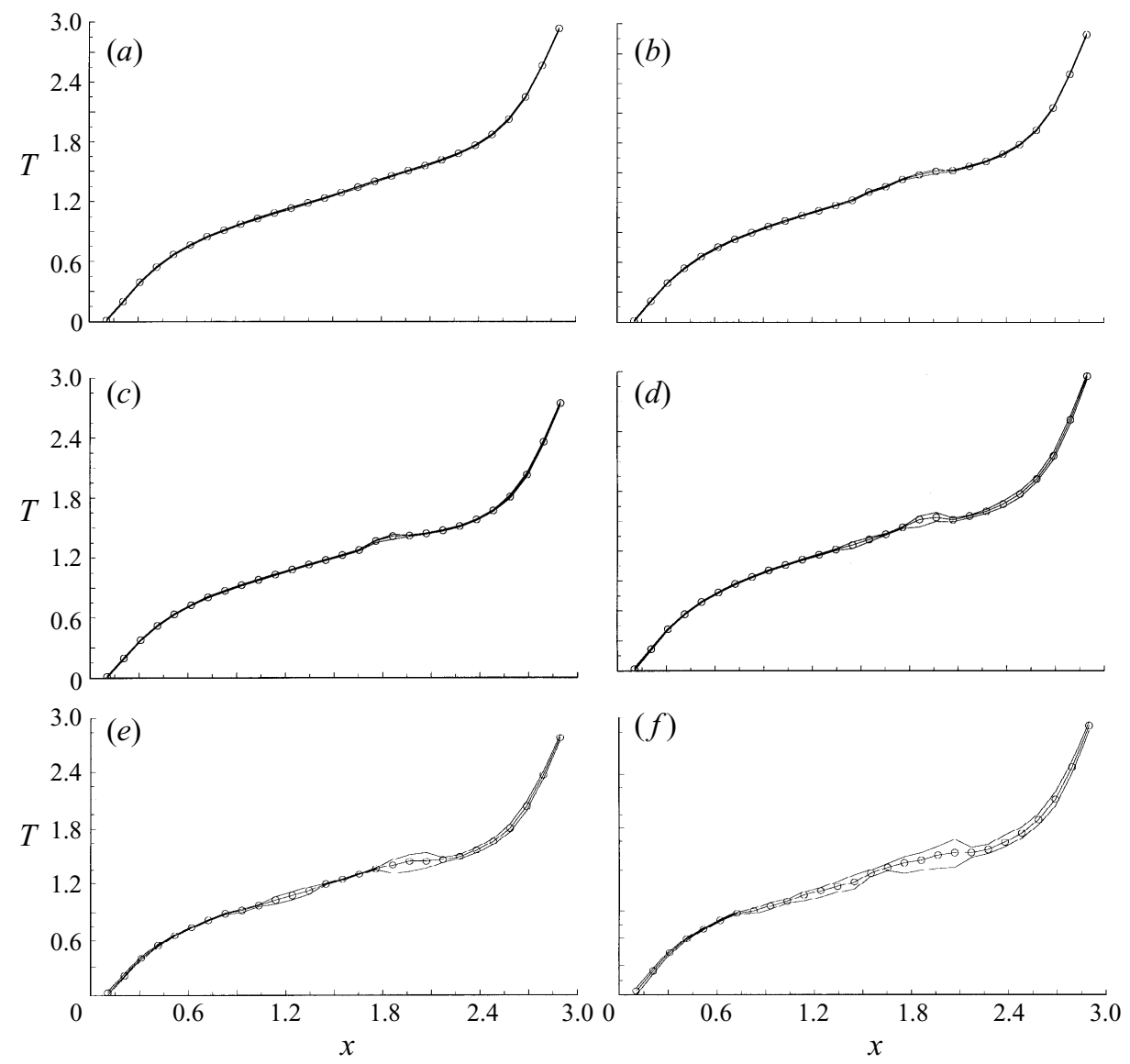

FIGURE 12. Experimentally measured horizontal temperature profiles for a sequence of Grashof numbers: $(a) G r=1.3 \times 10^{5},(b) G r=1.5 \times 10^{5},(c) G r=1.6 \times 10^{5},(d) G r=1.7 \times 10^{5}$, (e) $\mathrm{Gr}=1.8 \times 10^{5},(f) \mathrm{Gr}=1.9 \times 10^{5}$.

plot of the temperature is shown in figure 11(b). The temperature distribution displays an s-shaped vertical profile, and a very small symmetric bulge in the horizontal direction. The amplitude of the s-shape corresponds to $40 \%$ of the applied temperature difference and is consistent with the profiles used to compare with the Hadley-cell and numerical model in $\S 5.1$. The small, symmetric bulge in the horizontal direction is evidence of the damping effect of the sidewalls since, in the centre of the container, the fluid flow is faster and the heat transport stronger. However, this is a very weak effect since the amplitude of the bulge is $3 \%$ of the applied temperature difference, i.e. an order of magnitude smaller than the main convective flow. The flow field is left-right symmetric and does not show any strong three-dimensional features. However, more recent calculations by A. Juel (1997, personal communication) suggest that there are subtle three-dimensional effects present in the flow at all Grashof numbers and this is the subject of current research.

\subsection{Lengthwise flow structure}

We present a set of six lengthwise temperature profiles measured in the $1.00 \times 3.02 \times 1.00$ cell in figure 12. The aim of this particular investigation is to see if there is any evidence 
for multicellular flow as predicted by previous numerical studies. Each plot contains three lines, one each for the minimum, mean and maximum of the temperature measurements. The qualitative form of the profiles shown is noticeably different from the numerical ones presented in figure $2(b)$ since the pronounced flattening of the calculated profile at the midpoint is not seen in the experimental results. While our numerical results are for smaller Grashof number, increasing this parameter only increases the flattening of the profile and hence the qualitative divergence between the two sets of results increases.

Inspection of the profiles presented in figures $12(c, d)$ shows the development of distinct bumps in the profiles. These bumps are a result of significant variation in the value of the temperature at these positions with time and are indicators of localized oscillations in the flow. We believe that they correspond to regions of strong shear between co-rotating cells, suggesting that there is multicellular flow present. Braunsfurth (1994) obtains similar evidence for flows of this type when the lid is absent in the experiment, indicating that they are robust features. It is known that the presence of three-cell flows depends on both aspect ratio and Prandtl number as discussed in the introduction. However, our numerical work on two-dimensional geometries suggests that three-cell flows are not present for the Prandtl number and aspect ratio used here. Finally, we note that multicellular flows have not yet been found in three-dimensional numerical results.

\section{Conclusions}

We have made a careful comparison between the temperature profiles used to characterize the flow field in a sidewall convection experiment on liquid gallium, twodimensional numerical calculations and a one-dimensional analytic solution. This is the first systematic investigation of the steady-state flow in a cell containing a low-Prandtl-number fluid. At sufficiently low Grashof number there is surprisingly good quantitative agreement between the results of analytical theory, numerical calculation and experiment. As the Grashof number is increased, the endwalls begin to influence the flow and the two-dimensional numerical solution and the experimental results diverge from the analytical curve, but remain in good quantitative agreement with each other. At even higher Grashof numbers, the two-dimensional numerical solution and the experiment diverge suggesting that three-dimensional effects become important. Measurements of the crosswise temperature distributions indicate that the flow retains its midplane symmetry and does not break it in the manner suggested by Hurle et al. (1974). In addition, the experimentally obtained longitudinal profiles show evidence of three-cell flows which are a feature of some two-dimensional numerical investigations.

This work was supported by the Defence Research Agency at Malvern (M.G.B., A.J.) and by an EPSRC grant (grant no. GR/K41311) (ACS). The authors thank Dave Broomhead and Andrew Cliffe for many useful discussions and Keith Long for his technical expertise in manufacturing and maintaining the experiment. The authors also thank AEA Technology, Harwell for the use of the ENTWIFE code. We would also like to thank the referees for their careful reading of the original manuscript and many helpful suggestions. 
Afrid, M. \& Zebib, A. 1990 Oscillatory three-dimensional convection in rectangular cavities and enclosures. Phys. Fluids A 2, 1318-1327.

Brandes, E. A. \& BrooK, G. B. (Eds.) 1992 Smithells Metals Reference Book. 7th edn. Butterworth/Heinemann.

Braunsfurth, M. G. 1994 A study of side-wall convection in liquid gallium. D.Phil. thesis, Oxford.

BRAunsfurth, M. G. \& Mullin, T. 1996 An experimental study of oscillatory convection in liquid gallium. J. Fluid Mech. 327, 199-219.

Cliffe, K. A., Garratt, T. J. \& Spence, A. 1993 Eigenvalues of the discretised Navier-Stokes equation with application to the detection of Hopf bifurcation. Adv. Comput. Maths 1, 337356.

Cormack, D. E., Leal, L. G. \& Imberger, J. 1974 Natural convection in a shallow cavity with differentially heated end walls. Part 1. Asymptotic theory. J. Fluid Mech. 65, 209-229.

Crochet, M. J., Geyling, F. T. \& Schaftingen, J. J. van 1983 Numerical simulation of the horizontal Bridgman growth of a Gallium Arsenide crystal. J. Cryst. Growth 65, 166-172.

Filyand, M. A. \& SEmenova, E. I. 1968 Handbook of the Rare Elements, Vol. 1: Trace Elements and Light Elements. MacDonald Technical and Scientific, London.

GiLl, A. E. 1966 A theory of thermal oscillations in liquid metals. J. Fluid Mech. 64, 577-588.

Gill, A. E. 1974 The boundary-layer regime for convection in a rectangular cavity. J. Fluid Mech. 26, 515-536.

Gray, D. D. \& Giorgini, A. 1976 The validity of the Boussinesq approximation for liquids and gases. Intl J. Heat Mass Transfer 19, 545-551.

Hampel, C. A. 1954 Rare Metals Handbook. Reimhold, New York.

Hart, J. E. 1972 Stability of thin non-rotating Hadley circulations. J. Atmos. Sci. 29, 687-697.

Hart, J. E. 1983 A note on the stability of low Prandtl number Hadley circulations. J. Fluid Mech. 132, 271-281.

Hung, M. C. \& ANDERECK, C. D. 1988 Transitions in convection driven by a horizontal temperature gradient. Phys. Lett. A 132, 253-258.

Hurle, D. T. J., Jakeman, E. \& Johnson, C. P. 1974 Convective temperature oscillations in molten Gallium. J. Fluid Mech. 64, 565-576.

Iida, T. \& Guthrie, R. I. L. 1993 The Physical Properties of Liquid Metals. Clarendon.

Kaye, G. W. C. \& Laby, T. H. 1982 Tables of Physical and Chemical Constants, 14th edn. Longman.

Kuo, H. P. \& Korpella, S. A. 1988 Stability and finite amplitude natural convection in a shallow cavity with insulated top and bottom heated from the side. Phys. Fluids 31, 33-42.

LAURE, P. 1987 Study on convective motions in a rectangular cavity with horizontal gradient of temperature J. Méch. Théor. Appl. 6, 351-382.

March, N. H. 1968 Liquid Metals. Pergamon.

Metals Handbook 1990 10th edn, vol. 2. ASM.

MÜlleR, A. \& Wiehelm, M. 1964 Periodische Temperaturschwankungen in flüssigem InSb als Ursache schichtweisen Einbaus von Te in Kristallisierendes InSb. Z. Naturf. A 19, 254-263.

Pratte, J. M. \& Hart, J. E. 1990 Endwall driven, low Prandtl number convection in a shallow rectangular cavity. J. Cryst. Growth 102, 54-68.

Roux, B. (Ed.) 1990 GAMM Workshop Notes on Numerical Fluid Dynamics, vol. 27. Vieweg.

Skeldon, A. C., Riley, D. S. \& Cliffe, K. A. 1996 Convection in a low Prandtl number fluid. J. Cryst. Growth 162, 95-106.

Smithells, C. J. \& Brandes, E. A. 1976 Metals reference book, 5th edn. Butterworths.

Thevenard, D., Rouzaud, A., Comera, J. \& Favier, J. J. 1991 Influence of convective thermal oscillations on a solidification interface in Bridgeman growth. J. Cryst. Growth 108, 572-582.

Touloukian, Y. S., Powell, R. W., Ho, C. Y. \& Klemans, P. G. (Eds.) 1979 Thermophysical Properties of Matter, Vol. 1: Thermal Conductivity: Metallic Elements and Alloys. The TPRC Data Series, IFI/Plenum.

WeAst R. C. et al. 1993 CRC Handbook of Chemistry and Physics, 74th edn. CRC Press Inc.

WINTERS, K. H. 1988 Oscillatory convection in liquid metals in a horizontal temperature gradient. Intl J. Num. Meth. Engng NSI 401-414. 